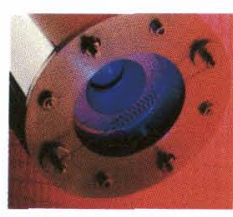

Industrial Productivity

\title{
Induction Heating Systems
}

More than a decade ago, Langley Research Center conducted research on a new method for in-space joining of plastic and composite components of space structures. A new method was needed because plastics and composites are difficult to join in the airless environment of

Induction space by conventional methods. Adhesive

beating bonding, for example, is not reliable in a vacuum,

systems

enable spot riveting techniques often deform the material, and mechanical fasteners require hole preparation and seam and special hardware.

Langley researchers

bonding of decided the best approach was induction

many heating, or magnetic heating, which causes plastics, little or no deformation and can be used with composites any type of thermoplastic material. They developed and patented a prototype system that had

and metals applicability not only in space structure assembly but also offered advantages in a variety of terrestrial applications in the automotive, appliance, aerospace and construction

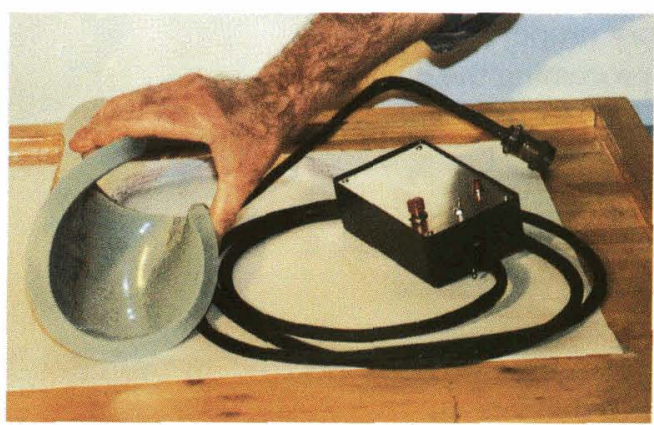
industries, plus military utility as a battlefield repair tool.

In 1981, Inductron Corporation, Grafton, Virginia, obtained an exclusive NASA license to use the induction heating technology in commercial applications.

Inductron initiated its own program to develop equipment and technology specifically for aircraft manufacture and repair. From that beginning, the company produced a series of induction heating systems and associated equipment — such as heating heads and joining tools - with applicability not only in aircraft manufacture/repair but also in a number of industrial and military applications. According to company literature, Inductron induction heating systems enable spot and seam bonding of many plastics, composites and metals in a fraction of the time required by conventional joining methods. A sampling of applications includes battlefield repair of aircraft windscreens, skins, hydraulic lines and rotor blades; rapid attachment of strain gauges; laboratory testing of adhesives; and manufacture and repair of composite assemblies.

Inductron produces several models of the Torobonder low-powered, self-tuning, portable induction bonding systems. Generally similar in size 


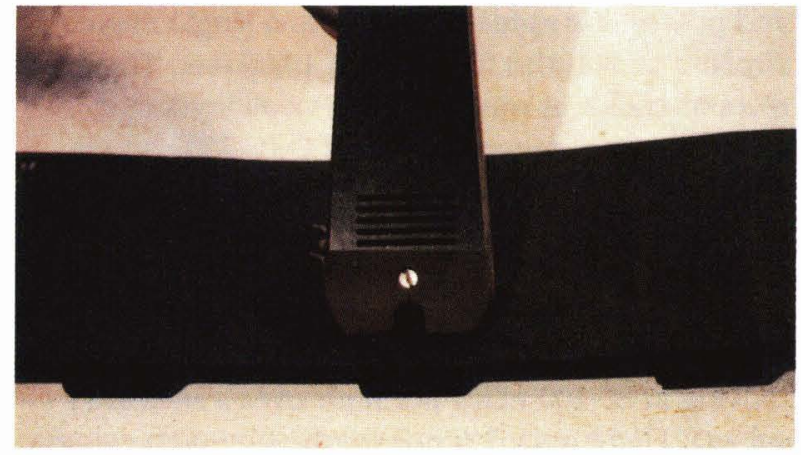

and weight (24-27 pounds), they differ primarily in output wattage. They come with a number of interchangeable heads such as the Torobonder Flex Head shown in closeup (lower left, opposite page) and in use (left) for repair of an aircraft windscreen. In the latter photo, the Flex Head is being fitted over a patch on the windscreen; the power supply delivers bonding heat and distributes it - on flat or curved surfaces - evenly throughout patch area.

Another Inductron development is the E Heating Head (above). Earlier methods for inductively heating two separate areas (spots or seams) require two separate heating operations. Inductron's E head can perform inductive heating of two spots or seams in a single operation. The unit offers advantages in aircraft manufacture/repair for attaching stiffeners to aircraft panels, and in automobile manufacture for attaching stiffener legs for strengthening door, trunk and hood panels.

Inductron has developed a Toroid Joining Gun applicable to heating a variety of conductive

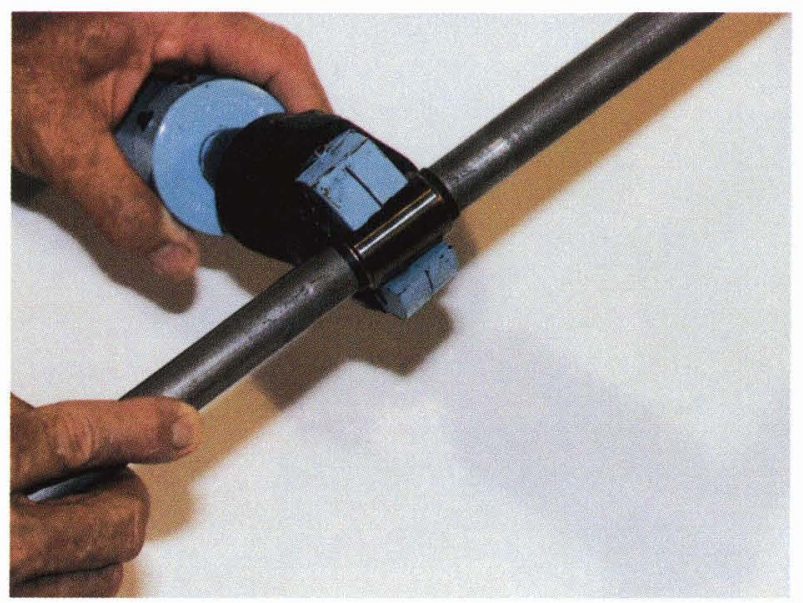

materials. One version of the gun is reconfigured for a special type of job important in military and industrial maintenance; heating metal heat-to-shrink couplings and fittings, typically for repair of hydraulic, air or plumbing lines. These shrink fittings are heated to a high temperature at which they shrink and bond to the line. In this application, the Inductron device offers advantages over earlier heatto-shrink methods in that it has no open flame and is non-hazardous; generates focused and controllable heat; does not adversely affect surrounding objects, such as wire harnesses or fuel lines; and has a long shelf life. At lower left, the tool is shown heating a coupling; the heating head comes in several sizes to accommodate a range of fitting and coupling diameters.

Shown below is still another Inductron system, the Torobrazer, which offers a new and advantageous method of brazing and annealing sawblade joints employing the induction heating process. Advantages cited include lightweight portability, low cost, low power, safety and ease of operation, even for inexperienced personnel.

All of these devices and others in the Inductron line stemmed from the original Langley induction heating research. In 1990, Inductron negotiated an agreement with NASA to patent all induction heatingrelated inventions in NASA's name, with Inductron granted exclusive rights to practice the technology.
All of these

devices

stemmed

from the

original

Langley

induction

beating

research

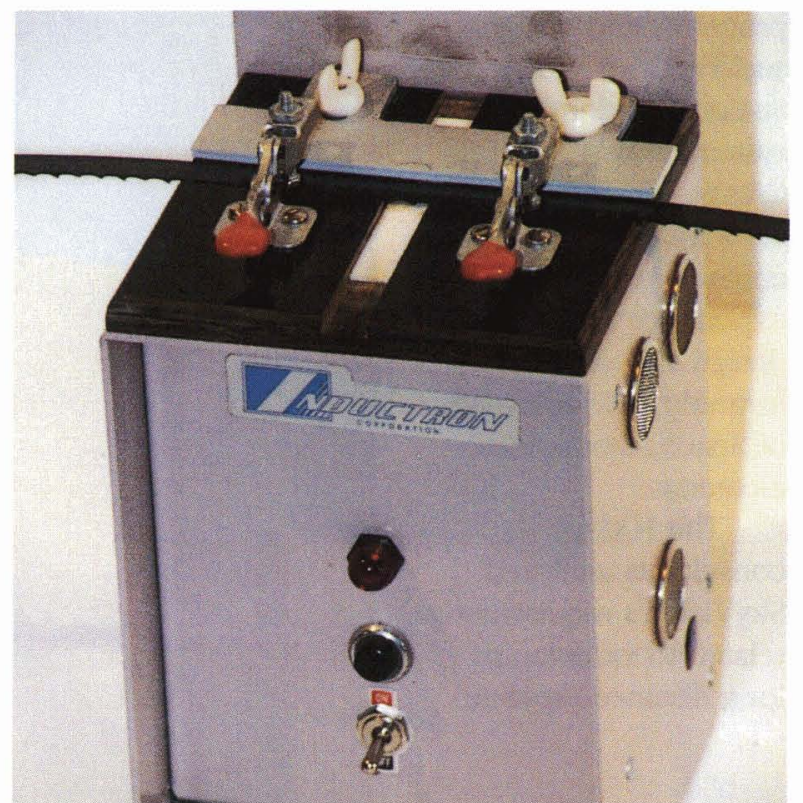

